\title{
Prognostic model of invasive ductal carcinoma of the breast based on differentially expressed glycolysis-related genes
}

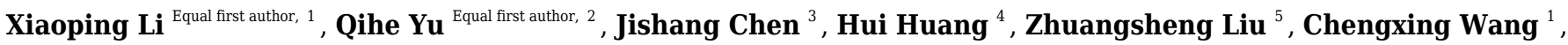 \\ Yaoming $\mathrm{He}^{1}{ }^{1}$, Xin Zhang ${ }^{6}$, Weiwen $\mathrm{Li}^{7}$, Chao $\mathrm{Li}^{1}{ }^{1}$, Jinglin Zhao ${ }^{1}$, Wansheng Long ${ }^{\text {Corresp. } 5}$ \\ ${ }^{1}$ Department of Gastrointestinal Surgery, Affiliated Jiangmen Hospital of Sun Yat-sen University, Jiangmen, Guangdong, China \\ Department of Oncology, Affiliated Jiangmen Hospital of Sun Yat-sen University, Jiangmen, Guangdong, China \\ 3 Department of Breast Surgery, Yangjiang people's Hospital, Yangjiang, Guangdong, China \\ 4 Department of Breast Surgery, Jiangmen Maternity \& Chile Health Care Hospital, Jiangmen, Guangdong, China \\ 5 Department of Radiology, Affiliated Jiangmen Hospital of Sun Yat-sen University, Jiangmen, Guangdong, China \\ 6 \\ ${ }^{6}$ Clinical Experimental Center, Jiangmen Key Laboratory of Clinical Biobanks and Translational Research, Affiliated Jiangmen Hospital of Sun Yat-sen \\ University, Jiangmen, Guangdong, China \\ 7 Department of Breast and Thyroid Surgery, Affiliated Jiangmen Hospital of Sun Yat-sen University, Jiangmen, Guangdong, China \\ Corresponding Author: Wansheng Long \\ Email address: jmlws2@163.com
}

Background: Invasive ductal carcinoma (IDC) is a common pathological type of breast cancer that is characterized by high malignancy and rapid progression. Upregulation of glycolysis is a hallmark of tumor growth, and correlates with the progression of breast cancer. We aimed to establish a model to predict the prognosis of patients with breast IDC based on differentially expressed glycolysis-related genes (DEGRGs) .

Methods: Transcriptome data and clinical data of patients with breast IDC were from The Cancer Genome Atlas (TCGA). Glycolysis-related gene sets and pathways were from the Molecular Signatures Database (MSigDB). DEGRGs were identified by comparison of tumor tissues and adjacent normal tissues. Univariate Cox regression and least absolute shrinkage and selection operator (LASSO) regression were used to screen for DEGRGs with prognostic value. A risk-scoring model based on DEGRGs related to prognosis was constructed. Receiver operating characteristic (ROC) analysis and calculation of the area under the curve (AUC) were used to evaluate the performance of the model. The model was verified in different clinical subgroups using an external dataset (GSE131769). A nomogram that included clinical indicators and risk scores was established. Gene function enrichment analysis was performed, and a protein-protein interaction network was developed.

Results: We analyzed data from 772 tumors and 88 adjacent normal tissues from the TCGA database and identified 286 glycolysis-related genes from the MSigDB. There were 185 DEGRGs. Univariate Cox regression and LASSO regression indicated that 13 of these genes were related to prognosis. A riskscoring model based on these 13 DEGRGs allowed classification of patients as high-risk or low-risk according to median score. The duration of overall survival (OS) was longer in the low-risk group $(\mathrm{P}<0.001)$, and the AUC was 0.755 for 3 -year OS and 0.726 for 5 -year OS. The results were similar when using the GEO data set for external validation (AUC for 3-year OS: 0.731, AUC for 5-year OS: 0.728). Subgroup analysis showed there were significant differences in OS among high-risk and low-risk patients in different subgroups (T1-2, T3-4, N0, N1-3, M0, TNBC, non-TNBC; all $P<0.01$ ). The C-index was 0.824 , and the AUC was 0.842 for 3 -year OS and 0.808 for 5 -year OS from the nomogram. Functional enrichment analysis demonstrated the DEGRGs were mainly involved in regulating biological functions.

Peer] reviewing PDF | (2020:07:50871:1:1:NEW 2 Oct 2020) 
Conclusions: Our prognostic model, based on 13 DEGRGs, had excellent performance in predicting the survival of patients with IDC of the breast. These DEGRGs appear to have important biological functions in the progression of this cancer. 
1 Prognostic model of invasive ductal carcinoma of the breast based on differentially expressed glycolysis-related genes

4 Xiaoping $\mathrm{Li}^{1 *}$, Qihe $\mathrm{Yu}^{2 *}$, Jishang Chen ${ }^{3}$, Hui Huang ${ }^{4}$, Zhuangsheng Liu ${ }^{5}$, Chengxing Wang ${ }^{1}$,

5 Yaoming $\mathrm{He}^{1}$, Xin Zhang 6 , Weiwen $\mathrm{Li}^{7}$, Chao $\mathrm{Li}^{1}$, Jinlin Zhao ${ }^{1 \#}$, Wansheng Long ${ }^{5 \#}$

$7{ }^{1}$ Department of Gastrointestinal Surgery, Affiliated Jiangmen Hospital of Sun Yat-sen University,

8 Jiangmen, Guangdong, China

9 2Department of Oncology, Affiliated Jiangmen Hospital of Sun Yat-sen University, Jiangmen,

10 Guangdong, China

$11{ }^{3}$ Department of Breast Surgery, Yangjiang People's Hospital, Yangjiang, Guangdong, China

$12{ }^{4}$ Department of Breast Surgery, Jiangmen Maternity \& Child Health Care Hospital, Jiangmen,

13 Guangdong, China

$14{ }^{5}$ Department of Radiology, Affiliated Jiangmen Hospital of Sun Yat-sen University, Jiangmen,

15 Guangdong, China

$16{ }^{6}$ Clinical Experimental Center, Jiangmen Key Laboratory of Clinical Biobanks and Translational

17 Research, Affiliated Jiangmen Hospital of Sun Yat-sen University, Jiangmen, Guangdong, China

$18{ }^{7}$ Department of Breast and Thyroid Surgery, Affiliated Jiangmen Hospital of Sun Yat-sen

19 University, Jiangmen, Guangdong, China

$21 *$ Equal contribution 


\section{Correspondence to:}

24 \#Wansheng Long (Department of Radiology, Affiliated Jiangmen Hospital of Sun Yat-sen

25 University, Jiangmen, Guangdong, China, E-mail: jmlws2@163.com)

26 \#Jinglin Zhao (Department of Gastrointestinal Surgery, Affiliated Jiangmen Hospital of Sun Yat-

27 sen University, Jiangmen, Guangdong, China, E-mail: 403612238@qqq.com)

40 Abstract

41 Background: Invasive ductal carcinoma (IDC) is a common pathological type of breast cancer

42 that is characterized by high malignancy and rapid progression. Upregulation of glycolysis is a 
43 hallmark of tumor growth, and correlates with the progression of breast cancer. We aimed to

44 establish a model to predict the prognosis of patients with breast IDC based on differentially

45 expressed glycolysis-related genes (DEGRGs).

46 Methods: Transcriptome data and clinical data of patients with breast IDC were from The

47 Cancer Genome Atlas (TCGA). Glycolysis-related gene sets and pathways were from the

48 Molecular Signatures Database (MSigDB). DEGRGs were identified by comparison of tumor

49 tissues and adjacent normal tissues. Univariate Cox regression and least absolute shrinkage and

50 selection operator (LASSO) regression were used to screen for DEGRGs with prognostic value.

51 A risk-scoring model based on DEGRGs related to prognosis was constructed. Receiver

52 operating characteristic (ROC) analysis and calculation of the area under the curve (AUC) were

53 used to evaluate the performance of the model. The model was verified in different clinical

54 subgroups using an external dataset (GSE131769). A nomogram that included clinical indicators

55 and risk scores was established. Gene function enrichment analysis was performed, and a

56 protein-protein interaction network was developed.

57 Results: We analyzed data from 772 tumors and 88 adjacent normal tissues from the TCGA

58 database and identified 286 glycolysis-related genes from the MSigDB. There were 185

59 DEGRGs. Univariate Cox regression and LASSO regression indicated that 13 of these genes

60 were related to prognosis. A risk-scoring model based on these 13 DEGRGs allowed

61 classification of patients as high-risk or low-risk according to median score. The duration of

62 overall survival (OS) was longer in the low-risk group $(\mathrm{P}<0.001)$, and the AUC was 0.755 for 3 -

63 year OS and 0.726 for 5-year OS. The results were similar when using the GEO data set for 
64

65

66

67

external validation (AUC for 3-year OS: 0.731, AUC for 5-year OS: 0.728). Subgroup analysis showed there were significant differences in OS among high-risk and low-risk patients in different subgroups (T1-2, T3-4, N0, N1-3, M0, TNBC, non-TNBC; all $P<0.01$ ). The C-index was 0.824 , and the AUC was 0.842 for 3 -year OS and 0.808 for 5 -year OS from the nomogram. Functional enrichment analysis demonstrated the DEGRGs were mainly involved in regulating biological functions.

Conclusions: Our prognostic model, based on 13 DEGRGs, had excellent performance in predicting the survival of patients with IDC of the breast. These DEGRGs appear to have important biological functions in the progression of this cancer.

Subjects: Bioinformatics, Oncology, Breast

Keywords: Breast invasive ductal carcinoma; glycolysis; miRNA; nomogram; functional enrichment analysis

\section{Introduction}

Breast invasive ductal carcinoma (IDC) is the most common malignant tumor in females worldwide(Harbeck et al., 2019; Hanker et al., 2020). In 2018, there were more than 266,000 cases of breast IDC among females in the United States, and this cancer accounted for $30 \%$ of malignant tumors in females, far more than lung cancer (13\%) (Bray et al., 2018; Ahmad, 2019). The prognosis of women with breast IDC is related to the activation or silencing of various 
85

86

87

88

89

90

biological functions in tumor tissues and signaling pathways. There are prognostic models based on tumor immunity and autophagy, but no models have exclusively focused on IDC ( Li et al., 2020; Zhang et al., 2019; Hu et al., 2020) and few models examined genes that function in basic metabolism.

Glycolysis is a series of reactions that catabolize most carbohydrates. "Metabolic reprogramming" is the hallmark of tumors, and glycolysis is the main source of energy for tumor cells, even when there is insufficient oxygen ( $W u$ et al., 2020a). Moreover, activation of glycolysis-related genes occurs in almost all tumor cells. For example, Dai et al. found that glycolysis promoted the progression of pancreatic cancer and induced gemcitabine chemotherapy resistance (Dai et al., 2020). Long noncoding RNAs (IncRNAs) that interact with Long Intergenic Noncoding RNA for IGF2BP2 Stability (LINRIS) activate aerobic glycolysis in tumor cells, and can affect the development and prognosis of rectal cancer (Wang et al., 2019).

Research on the function of glycolysis-related genes in breast tumors showed that hexokinase (HK2) had high expression in breast IDC cells, and that HK2 silencing inhibited IDC (Patra et al., 2013; Cao et al., 2020). Other research showed that overexpression of 6Phosphofructo 2-kinase/fructose 2, 6-bisphosphatase 3 (PFKFB3) promoted the progression of breast IDC, and had negative associations with progression-free survival (PFS), distant metastasis-free survival (DMFS), and overall survival (OS) in patients with breast IDC ( $O^{\prime} \mathrm{Neal}$ et al., 2016; Peng et al., 2018). Thus, glycolysis-related genes have a potentially significant impact on the progression of breast tumors and on the survival and prognosis of patients with breast tumors. 

and determine the potential functions of glycolysis-related genes in the progression of breast IDC.

\section{Materials and methods}

\section{Data resources and preprocessing}

The transcriptome data and corresponding clinical data of breast invasive ductal carcinoma were downloaded from the TCGA database (https://www.cancer.gov/) (Tomczak et al., 2015). The data set of glycolysis-related genes was obtained from the MSigDB database (http://www.hmdb.ca). Using $\left|\log _{2} \mathrm{FC}\right|>0.5$ and false discovery rate (FDR) $<0.05$ as the cut-off value, the data was normalized with the "edgeR" package from $\mathrm{R}$, and then the differential analysis was performed to obtain the differential expression of glycolysis-related genes (DEGRGs) between the tumor tissue and normal tissue.

\section{Construction of risk-scoring model}

Based on the above DGRG, univariate Cox regression and LASSO regression were used to screen out prognostic-related glycolysis genes. The risk score was evaluated by the coefficient of each prognostic-related glycolysis gene. The risk scoring formula was constructed as

Risk scores $=\sum_{1}^{i}$ (coefi $*$ expri), where $\mathrm{i}$ is the number of genes used to build the model, coefi is the coefficients of the genes in the model, and expriis the expression of genes in the model. Taking the median risk score as the cut-off point, patients were divided into high-risk and low-risk groups. The survival outcome of the two groups was observed by Kaplan-Meier survival analysis. 
126 Receiver operating characteristic (ROC) curve was applied to calculate the area under the curve

127 (AUC) to evaluate the predictive ability of the risk-scoring model. Independent GEO

128 (https://www.ncbi.nlm.nih.gov/geo/) data sets are used to verify the above results (Barrett et al.,

129 2013). Univariate Cox regression and multivariate Cox regression were used to identified the

130 independent prognostic factors among risk scores, age, tumor TNM stage, and whether triple

131 negative breast cancer (TNBC) or not. Through clinical survival analysis, the predictive ability

132 of the risk-scoring model in different clinical subgroups was clarified.

133

134

135

136

137

138

139

140

141

\section{External validation of the risk scoring model}

The TCGA results were validated using the GEO (https:/www.ncbi.nlm.nih.gov/geo/) dataset (GSE131769). For this validation, the outcomes of the two groups were compared using Kaplan-Meier survival analysis. ROC curves were used to calculate AUCs and evaluate the predictive performance of the risk-scoring model.

\section{Construction of the nomogram}

A nomogram was constructed based on the results of the multivariate Cox regression, with clinical information such as age, TMN stage, TNBC status, and DEGRG risk scores, to predict 3year and 5-year OS. The predictive ability of the nomogram was evaluated by calculating the Cindex and the calibration chart, clinical decision curve analysis, and an ROC curve.

\section{Function enrichment analysis}

We analyzed the genes that were differentially expressed in the high-risk and low-risk groups using the Kyoto Encyclopedia of Genes and Genomes (KEGG) and Gene Ontology (GO) to identify pathway enrichment (Kanehisa et al., 2019). This analysis allowed identification of the 
147 functions of the differentially expressed genes. We then examined whether the differentially expressed genes were involved in the development of breast cancer.Construction of interactive

network diagram

To determine the relationship of the DEGRG model with prognosis, a network between genes was developed. The prognosis-related genes were imported into Search Tool for the

Retrieval of Interacting Genes Proteins (STRING) to construct an interaction network

153 (Szklarczyk et al., 2019).

\section{Results}

\section{DEGRGs in breast IDC}

We obtained gene expression data and clinical data of females with IDC of the breast (772

tumor tissues, 88 adjacent normal tissues) from the TCGA database and the glycolysis gene set (286 genes) from the GSEA website. Based on standard cut-off values for fold-change in gene expression $\left(\left|\log _{2}(\mathrm{FC})\right|>0.5\right)$ and false discovery rate $(\mathrm{FDR}<0.05)$, the IDC tissues had 185 DEGRGs, with 67 down-regulated genes and 118 up-regulated genes (Figure 1A, B,

Supplementary material Table1).

\section{Relationship of DEGRGs with prognosis and risk-scoring model}

We then identified patients with follow-up times greater than 30 days, and performed univariate Cox regression and LASSO regression to screen for DEGRGs that were related to prognosis (Figure 2A, B, C). This analysis indicated that 13 DEGRGs were closely related to prognosis. 
patients into high-risk and low-risk groups based on median risk score. Kaplan-Meier survival

analysis showed that patients with high-risk had significantly reduced duration of OS $(P=9.795$

$\times 10^{-8}$, Figure 3A). ROC analysis indicated the AUC was 0.755 for 3 -year OS and 0.726 for 5-

year OS (Figure 3B). The risk curve and scatterplot (Figure 3C, D) show the risk scores and

survival status of all patients, and indicate that the risk coefficient and mortality rate were greater

in the high-risk group. We plotted a "heat map" to visualize the expression of the 13 DEGRGs in

the high-risk and low-risk groups (Figure 3E). Taken together, these results confirm that 13

DEGRGs were significant prognostic indicators for patients with IDC of the breast.

We performed univariate and multivariate Cox regression to evaluate the effect of risk score,

age, triple-negative breast cancer (TNBC), and TNM stage on patient prognosis (Figures 4A, B).

The results confirmed that the risk score was an independent prognostic factor for patients with

IDC of the breast (adjusted hazard ratio: $2.71,95 \%$ confidence interval: $1.87-3.94$ ).

We also performed survival analysis of different subgroups based on TNM status (Figure 5).

This analysis indicated that the risk-scoring model had good predictive value in the T1-2

subgroup, T3-4 subgroup, N0 subgroup, N1-3 subgroup, M0 subgroup, TNBC subgroup, and

non-TNBC subgroup (all $P<0.001)$, but not in the M1 subgroup $(P=0.857)$.

185

186

187

\section{External validation of the risk scoring model}

We verified the model using the GEO dataset (GSE131769). Kaplan-Meier survival curves showed that patients with high-risk had a significantly shorter duration of OS $\left(P=3.245 \times 10^{-3}\right.$, 
188 189 190

Figure 6A). ROC analysis indicated that the AUC was 0.731 for 3 -year OS and was 0.728 for 5year OS (Figure 6B). These results confirm the validity of our risk scoring model.

\section{Construction of the prediction model}

Based on the results of the multivariate Cox regression, we developed a nomogram based on age, TMN stage, risk score, and TNBC status to predict 3-year and 5-year OS (Figure 7A). We then used the C-index, clinical decision curve, calibration chart, and ROC curve to evaluate the predictive performance of the nomogram (Figure 7B-F). The results indicated that the prognostic model had good prediction accuracy, with a C-index of 0.824, an AUC for 3-year OS of 0.842 , and an AUC for 5-year OS of 0.808 . These results verified the predictive ability of the nomogram.

\section{Gene function enrichment analysis}

To explore the potential mechanisms of prognosis-related DEGRGs in breast invasive ductal carcinoma, we performed KEGG enrichment analysis and GO enrichment analysis.

KEGG pathway enrichment analysis showed that the gene set was enriched in the cell cycle,

DNA replication, glycolysis and gluconeogenesis, RNA degradation, arachidonic acid metabolism, cytokine-cytokine receptor interactions, cytosolic DNA sensing, and ribosome function (Figure 8A-H). GO enrichment analysis showed that the gene set was enriched in the cell cycle G1-S phase transition, DNA geometric changes, the meiotic cell cycle process, regulation of cellular response to heat, cytokine-mediated signaling pathways, regulation of homotypic cell-cell adhesion, regulation of production of molecular mediators of immune responses, and $\mathrm{T}$ cell differentiation (Figure 8I-P). 
209

210

211

212

213

214

215

216

217

218

219

220

221

222

223

224

225

226

227

228

229

\section{Construction of an interactive network diagram}

We constructed a network diagram to visualize the interactions between hub genes and different miRNAs to better understand the potential functions of the different DEGRGs on prognosis. The results from STRING showed there were 6 interacting hub genes: P4HA2, P4HA1, PGK1, G6PD, HK3, and PMM2 (Figure 9).

\section{Discussion}

Breast IDC is the most common pathological type of breast tumor, and morbidity and mortality from this cancer continue to increase (Harbeck et al., 2019; Badve \& Gokmen-Polar, 2019). There is evidence that changes in glycolysis-related genes have multiple effects on the prognosis of these patients (Li et al., 2020; Chen et al., 2019). In particular, tumor cells reprogram the glycolysis pathway to accommodate the increased energy required for malignant transformations, including invasion and metastasis (Shen et al., 2020; Abbaszadeh et al., 2020). Given the importance of glycolysis on tumor prognosis, numerous research groups have developed models based on glycolysis-related genes in their studies of different cancers (Zhang et al., 2019; Wang et al., 2019; Karasinska et al., 2020). However, no previous study developed a prognostic model for patients with breast IDC based on glycolysis-related genes.

In this research, we identified 13 DEGRGs that were related to prognosis in patients with breast IDC, and then established a risk-scoring model. The results showed that the OS of patients in the high-risk group was significantly shorter than that of patients in the low-risk group. We 
230 also examined the impact of patient age, TMN stage, TNBC status, and risk score to construct a

231 nomogram that predicts the 3-year and 5-year OS of these patients. Our application of various

232 evaluation methods indicated that the nomogram had good performance in the prediction of OS.

233 Our ROC analysis showed that the AUC was 0.842 for 3-year OS and 0.808 for 5-year OS,

234 higher than the AUC values reported in previous models (Lin et al., 2020; Xie et al., 2019), thus indicating that our model had better predictive ability.

Among the 13 DEGRGs we used to construct the risk model, increased levels of $\mathrm{P} 4 \mathrm{H} 2 \mathrm{~A}$,

NUP155, ALDH3B1, SDC1, G6PD, COPB2, B3GNT3, PMM2, and PGK1 were associated with poor prognosis and increased levels of HK3, AGRN, P4HA1, and ISG20 were associated with favorable prognosis (Supplementary material Table2). Previous research reported increased levels of P4HA2 and P4HA1 (the two isomers of collagen prolyl 4-hydroxylase) in several types of human cancers and that both enzymes promoted glycolysis in tumor cells (Li et al., 2019). PGK1 is the first key enzyme to produce ATP in the glycolytic pathway, PGK1 is not only a metabolic enzyme but also a protein kinase, which mediates the tumor growth, migration and invasion through phosphorylation some important substrates ( $F u \& Y u, 2020)$. There is also evidence that SDC1 can promote the migration of breast cancer cells across the blood-brain barrier by regulating the expression of cytokines, thus promoting brain metastasis (Sayyad et al., 2019). Mele et al. found that the overexpression of G6PD can induce lapatinib resistance in breast cancer and also found a significant correlation between high expression of G6PD and tumor recurrence (Mele et al., 2019). Sauter et al. found that the level of HK3 in the nipple 
251

252

253

254

255

256

257

258

259

260

261

262

263

264

265

266

267

268

269

270

271

considered an elevated HK3 level as a possible sign of early breast cancer (Mannello \&

Gazzanelli, 2001). Thus, these previous studies are consistent with our finding that glycolysisrelated genes were closely related to the occurrence and development of breast cancer and the prognosis of patients.

To further characterize the potential roles of the 13 DEGRGs in our risk model, we performed GO and KEGG enrichment analysis. The results showed that the gene set was enriched in cell cycle, DNA replication, glycolysis gluconeogenesis, RNA degradation, arachidonic acid metabolism, cytokine cytokine receptor interaction, cytosolic DNA sensing pathway and ribosome. Previous studies showed that under hypoxic conditions, metabolic reprogramming of breast tumor stem cells helped to maintain their growth and proliferation (Peng et al., 2018). Breast cancer occurs in patients with impaired immune function, in which cytokines function as growth signals for tumor cells. Many studies showed that interactions between the immune system and cancer cells, which are mediated by cytokines and chemokines, affect the initiation and progression of breast cancer and the response to treatment (Lim et al., 2018; King et al., 2017; Fabre et al., 2018). DNA replication is a fundamental biological process, and replication disorders can lead to genomic instability, an important feature of breast cancer. Many experimental and clinical studies have identified disorders of DNA replication during the development and progression of breast cancer (Kitao et al., 2018). Aerobic glycolysis pathway includes hexokinase, phosphofructokinase (PFK), and other genes (Wu et al., 2020b). These previous findings therefore support our conclusion that the 13 glycolysis-related genes identified here play an important role in the progression of breast tumors. 

mechanisms.

\section{Conclusions}

In conclusion, our prediction model, which is based on 13 DEGRGs and the clinical

DEGRGs and several related miRNAs thus appear to play an important role in the development and progression of breast IDC.

\section{Additional information and statements}

\section{Funding}

This study was supported by the Guangdong Medical Research Foundation (Grant No.

A2020622) and the Elite Young Scholars Program of Jiangmen Central Hospital (Grant No.

J201905). The fund sponsors had no role in the study design, data collection and analysis,

decision to publish, or preparation of the manuscript.

\section{Grant Disclosures}

The following grant information was disclosed by the authors:

Guangdong Medical Research Foundation: A2020622. 


\section{Competing Interests}

294 The authors declare that they have no competing interests.

295

296

297

298

299

300

301

302

303

304

305

306

307

308

309

310

311

312

313

314

315

316

317

318

\section{Author Contributions}

Xiaoping Li conceived the study, performed the data analysis, prepared the figures and tables, authored and reviewed drafts of the paper, and approved the final draft.

Qihe $\mathrm{Yu}$ collected the data from database, prepared the figures, reviewed drafts of paper, and approved the final draft.

Jinglin Zhao conceived the study, prepared the figures, reviewed drafts of the paper, and approved the final draft.

Wansheng Long conceived the study, prepared the figures, reviewed drafts of the paper, and approved the final draft.

\section{Data Availability}

The following information was supplied regarding data availability:

The RNA-seq transcriptome data and corresponding clinicopathological information of the data were obtained from TCGA: TCGA-BRCA.

\section{References:}

Harbeck, N, Penault-Llorca, F, Cortes, J, Gnant, M, Houssami, N, Poortmans, P, Ruddy, K, Tsang, J, Cardoso, F. 2019. Breast cancer. Nat Rev Dis Primers 5(1): 66 DIO 10.1038/s41572-019-0111-2.

Hanker, A B, Sudhan, D R, Arteaga, C L. 2020. Overcoming Endocrine Resistance in Breast Cancer. Cancer Cell 37(4): 496-513 DIO 10.1016/j.ccell.2020.03.009.

Bray, F, Ferlay, J, Soerjomataram, I, Siegel, R L, Torre, L A, Jemal, A. 2018. Global cancer statistics 2018: GLOBOCAN estimates of incidence and mortality worldwide for 36 cancers in 185 countries. CA Cancer J Clin 68(6): 394-424 DIO 10.3322/caac.21492.

Ahmad, A. 2019. Breast Cancer Statistics: Recent Trends. Adv Exp Med Biol 1152(1-7 DIO 10.1007/978-3-03020301-6_1. 
Li, B, Geng, R, Wu, Q, Yang, Q, Sun, S, Zhu, S, Xu, Z, Sun, S. 2020. Alterations in Immune-Related Genes as Potential Marker of Prognosis in Breast Cancer. Front Oncol 10(333 DIO 10.3389/fonc.2020.00333.

Zhang, L, Zhang, Z, Yu, Z. 2019. Identification of a novel glycolysis-related gene signature for predicting metastasis and survival in patients with lung adenocarcinoma. $J$ Transl Med 17(1): 423 DIO 10.1186/s12967019-02173-2.

Hu, D, Jiang, L, Luo, S, Zhao, X, Hu, H, Zhao, G, Tang, W. 2020. Development of an autophagy-related gene expression signature for prognosis prediction in prostate cancer patients. J Transl Med 18(1): 160 DIO 10.1186/s12967-020-02323-x.

Wu, Z, Wu, J, Zhao, Q, Fu, S, Jin, J. 2020a. Emerging roles of aerobic glycolysis in breast cancer. Clin Transl Oncol 22(5): 631-646 DIO 10.1007/s12094-019-02187-8.

Dai, S, Peng, Y, Zhu, Y, Xu, D, Zhu, F, Xu, W, Chen, Q, Zhu, X, Liu, T, Hou, C, Wu, J, Miao, Y. 2020. Glycolysis promotes the progression of pancreatic cancer and reduces cancer cell sensitivity to gemcitabine. Biomed Pharmacother 121(109521 DIO 10.1016/j.biopha.2019.109521.

Wang, Y, Lu, J H, Wu, Q N, Jin, Y, Wang, D S, Chen, Y X, Liu, J, Luo, X J, Meng, Q, Pu, H Y, Wang, Y N, Hu, P S, Liu, Z X, Zeng, Z L, Zhao, Q, Deng, R, Zhu, X F, Ju, H Q, Xu, R H. 2019. LncRNA LINRIS stabilizes IGF2BP2 and promotes the aerobic glycolysis in colorectal cancer. Mol Cancer 18(1): 174 DIO 10.1186/s12943-019-1105-0.

Patra, K C, Wang, Q, Bhaskar, P T, Miller, L, Wang, Z, Wheaton, W, Chandel, N, Laakso, M, Muller, W J, Allen, E L, Jha, A K, Smolen, G A, Clasquin, M F, Robey, B, Hay, N. 2013. Hexokinase 2 is required for tumor initiation and maintenance and its systemic deletion is therapeutic in mouse models of cancer. Cancer Cell 24(2): 213-228 DIO 10.1016/j.ccr.2013.06.014.

Cao, L, Wang, M, Dong, Y, Xu, B, Chen, J, Ding, Y, Qiu, S, Li, L, Karamfilova, Z E, Zhou, X, Xu, Y. 2020. Circular RNA circRNF20 promotes breast cancer tumorigenesis and Warburg effect through miR-487a/HIF1alpha/HK2. Cell Death Dis 11(2): 145 DIO 10.1038/s41419-020-2336-0.

O'Neal, J, Clem, A, Reynolds, L, Dougherty, S, Imbert-Fernandez, Y, Telang, S, Chesney, J, Clem, B F. 2016. Inhibition of 6-phosphofructo-2-kinase (PFKFB3) suppresses glucose metabolism and the growth of HER2+ breast cancer. Breast Cancer Res Treat 160(1): 29-40 DIO 10.1007/s10549-016-3968-8.

Peng, F, Li, Q, Sun, J Y, Luo, Y, Chen, M, Bao, Y. 2018. PFKFB3 is involved in breast cancer proliferation, migration, invasion and angiogenesis. Int J Oncol 52(3): 945-954 DIO 10.3892/ijo.2018.4257.

Tomczak, K, Czerwinska, P, Wiznerowicz, M. 2015. The Cancer Genome Atlas (TCGA): an immeasurable source of knowledge. Contemp Oncol (Pozn) 19(1A): A68-A77 DIO 10.5114/wo.2014.47136.

Barrett, T, Wilhite, S E, Ledoux, P, Evangelista, C, Kim, I F, Tomashevsky, M, Marshall, K A, Phillippy, K H, Sherman, P M, Holko, M, Yefanov, A, Lee, H, Zhang, N, Robertson, C L, Serova, N, Davis, S, Soboleva, A. 2013. NCBI GEO: archive for functional genomics data sets--update. Nucleic Acids Res 41(Database issue): D991-D995 DIO 10.1093/nar/gks1193.

Kanehisa, M, Sato, Y, Furumichi, M, Morishima, K, Tanabe, M. 2019. New approach for understanding genome variations in KEGG. Nucleic Acids Res 47(D1): D590-D595 DIO 10.1093/nar/gky962.

Szklarczyk, D, Gable, A L, Lyon, D, Junge, A, Wyder, S, Huerta-Cepas, J, Simonovic, M, Doncheva, N T, Morris, J H, Bork, P, Jensen, L J, Mering, C V. 2019. STRING v11: protein-protein association networks with increased coverage, supporting functional discovery in genome-wide experimental datasets. Nucleic Acids Res 47(D1): D607-D613 DIO 10.1093/nar/gky1131.

Peer] reviewing PDF | (2020:07:50871:1:1:NEW 2 Oct 2020) 
Badve, S S, Gokmen-Polar, Y. 2019. Ductal carcinoma in situ of breast: update 2019. Pathology 51(6): 563-569 DIO 10.1016/j.pathol.2019.07.005.

Li, W, Xu, M, Li, Y, Huang, Z, Zhou, J, Zhao, Q, Le K, Dong, F, Wan, C, Yi, P. 2020. Comprehensive analysis of the association between tumor glycolysis and immune/inflammation function in breast cancer. $J$ Transl Med 18(1): 92 DIO 10.1186/s12967-020-02267-2.

Chen, F, Chen, J, Yang, L, Liu, J, Zhang, X, Zhang, Y, Tu, Q, Yin, D, Lin, D, Wong, P P, Huang, D, Xing, Y, Zhao, J, Li, M, Liu, Q, Su, F, Su, S, Song, E. 2019. Extracellular vesicle-packaged HIF-1alpha-stabilizing lncRNA from tumour-associated macrophages regulates aerobic glycolysis of breast cancer cells. Nat Cell Biol 21(4): 498-510 DIO 10.1038/s41556-019-0299-0.

Shen, C, Xuan, B, Yan, T, Ma, Y, Xu, P, Tian, X, Zhang, X, Cao, Y, Ma, D, Zhu, X, Zhang, Y, Fang, J Y, Chen, H, Hong, J. 2020. m(6)A-dependent glycolysis enhances colorectal cancer progression. Mol Cancer 19(1): 72 DIO 10.1186/s12943-020-01190-w.

Abbaszadeh, Z, Cesmeli, S, Biray, A C. 2020. Crucial players in glycolysis: Cancer progress. Gene 726(144158 DIO 10.1016/j.gene.2019.144158.

Wang, Z H, Zhang, Y Z, Wang, Y S, Ma, X X. 2019. Identification of novel cell glycolysis related gene signature predicting survival in patients with endometrial cancer. Cancer Cell Int 19(296 DIO 10.1186/s12935-019-1001-0.

Karasinska, J M, Topham, J T, Kalloger, S E, Jang, G H, Denroche, R E, Culibrk, L, Williamson, L M, Wong, H L, Lee, M, O'Kane, G M, Moore, R A, Mungall, A J, Moore, M J, Warren, C, Metcalfe, A, Notta, F, Knox, J J, Gallinger, S, Laskin, J, Marra, M A, Jones, S, Renouf, D J, Schaeffer, D F. 2020. Altered Gene Expression along the Glycolysis-Cholesterol Synthesis Axis Is Associated with Outcome in Pancreatic Cancer. Clin Cancer Res 26(1): 135-146 DIO 10.1158/1078-0432.CCR-19-1543.

Lin, Q G, Liu, W, Mo, Y Z, Han, J, Guo, Z X, Zheng, W, Wang, J W, Zou, X B, Li, A H, Han, F. 2020. Development of prognostic index based on autophagy-related genes analysis in breast cancer. Aging (Albany NY) 12(2): 1366-1376 DIO 10.18632/aging.102687.

Xie, P, Ma, Y, Yu, S, An, R, He, J, Zhang, H. 2019. Development of an Immune-Related Prognostic Signature in Breast Cancer. Front Genet 10(1390 DIO 10.3389/fgene.2019.01390.

Li, Q, Wang, Q, Zhang, Q, Zhang, J, Zhang, J. 2019. Collagen prolyl 4-hydroxylase 2 predicts worse prognosis and promotes glycolysis in cervical cancer. Am J Transl Res 11(11): 6938-6951 DIO

Fu, Q, Yu, Z. 2020. Phosphoglycerate kinase 1 (PGK1) in cancer: A promising target for diagnosis and therapy. Life Sci 256(117863 DIO 10.1016/j.lfs.2020.117863.

Sayyad, M R, Puchalapalli, M, Vergara, N G, Wangensteen, S M, Moore, M, Mu, L, Edwards, C, Anderson, A, Kall, S, Sullivan, M, Dozmorov, M, Singh, J, Idowu, M O, Koblinski, J E. 2019. Syndecan-1 facilitates breast cancer metastasis to the brain. Breast Cancer Res Treat 178(1): 35-49 DIO 10.1007/s10549-019-05347-0.

Mele, L, la Noce, M, Paino, F, Regad, T, Wagner, S, Liccardo, D, Papaccio, G, Lombardi, A, Caraglia, M, Tirino, V, Desiderio, V, Papaccio, F. 2019. Glucose-6-phosphate dehydrogenase blockade potentiates tyrosine kinase inhibitor effect on breast cancer cells through autophagy perturbation. J Exp Clin Cancer Res 38(1): 160 DIO 10.1186/s13046-019-1164-5.

Mannello, F, Gazzanelli, G. 2001. Prostate-specific antigen (PSA/hK3): a further player in the field of breast cancer diagnostics? Breast Cancer Res 3(4): 238-243 DIO 10.1186/bcr302.

Peng, F, Wang, J H, Fan, W J, Meng, Y T, Li, M M, Li, T T, Cui, B, Wang, H F, Zhao, Y, An, F, Guo, T, Liu, X F, Zhang, L, Lv, L, Lv, D K, Xu, L Z, Xie, J J, Lin, W X, Lam, E W, Xu, J, Liu, Q. 2018. Glycolysis 
401

402

403

404

405

406

407

408

409

410

411

412

413

414

415

416

417

418

419

420

421

422

423

424

gatekeeper PDK1 reprograms breast cancer stem cells under hypoxia. Oncogene 37(8): 1062-1074 DIO 10.1038/onc.2017.368.

King, J, Mir, H, Singh, S. 2017. Association of Cytokines and Chemokines in Pathogenesis of Breast Cancer. Prog Mol Biol Transl Sci 151(113-136 DIO 10.1016/bs.pmbts.2017.07.003.

Fabre, J, Giustinniani, J, Garbar, C, Merrouche, Y, Antonicelli, F, Bensussan, A. 2018. The Interleukin-17 Family of Cytokines in Breast Cancer. Int J Mol Sci 19(12): DIO 10.3390/ijms19123880.

Lim, B, Woodward, W A, Wang, X, Reuben, J M, Ueno, N T. 2018. Inflammatory breast cancer biology: the tumour microenvironment is key. Nat Rev Cancer 18(8): 485-499 DIO 10.1038/s41568-018-0010-y.

Kitao, H, Iimori, M, Kataoka, Y, Wakasa, T, Tokunaga, E, Saeki, H, Oki, E, Maehara, Y. 2018. DNA replication stress and cancer chemotherapy. Cancer Sci 109(2): 264-271 DIO 10.1111/cas.13455.

Wu, Z, Wu, J, Zhao, Q, Fu, S, Jin, J. 2020b. Emerging roles of aerobic glycolysis in breast cancer. Clin Transl Oncol 22(5): 631-646 DIO 10.1007/s12094-019-02187-8.

Du Y, Lin, Y, Yin, K, Zhou, L, Jiang, Y, Yin, W, Lu, J. 2019. Single nucleotide polymorphisms of let-7-related genes increase susceptibility to breast cancer. Am J Transl Res 11(3): 1748-1759 DIO

Mi, Y, Liu, F, Liang, X, Liu, S, Huang, X, Sang, M, Geng, C. 2019. Tumor suppressor let-7a inhibits breast cancer cell proliferation, migration and invasion by targeting MAGE-A1. Neoplasma 66(1): 54-62 DIO 10.4149/neo_2018_180302N146.

Wang, W, Zhang, L, Wang, Y, Ding, Y, Chen, T, Wang, Y, Wang, H, Li, Y, Duan, K, Chen, S, Yang, Q, Chen, C. 2017. Involvement of miR-451 in resistance to paclitaxel by regulating YWHAZ in breast cancer. Cell Death Dis 8(10): e 3071 DIO 10.1038/cddis.2017.460.

Liu, C, Xing, H, Luo, X, Wang, Y. 2020. MicroRNA-342 targets Cofilin 1 to suppress the growth, migration and invasion of human breast cancer cells. Arch Biochem Biophys 687(108385 DIO 10.1016/j.abb.2020.108385.

Peer) reviewing PDF | (2020:07:50871:1:1:NEW 2 Oct 2020) 
Figure 1

Differentially expressed glycolysis-related genes between breast invasive ductal carcinoma and normal tissues.

(A) The volcano gram showed that compared with normal tissues, 67 GRGs were down-

regulated and 118 GRGs were up-regulated in breast invasive ductal carcinoma. $(P<0.05)(B)$ The heat map showed the expression of 185 DGRGs in both tumor tissues and normal tissues.

A
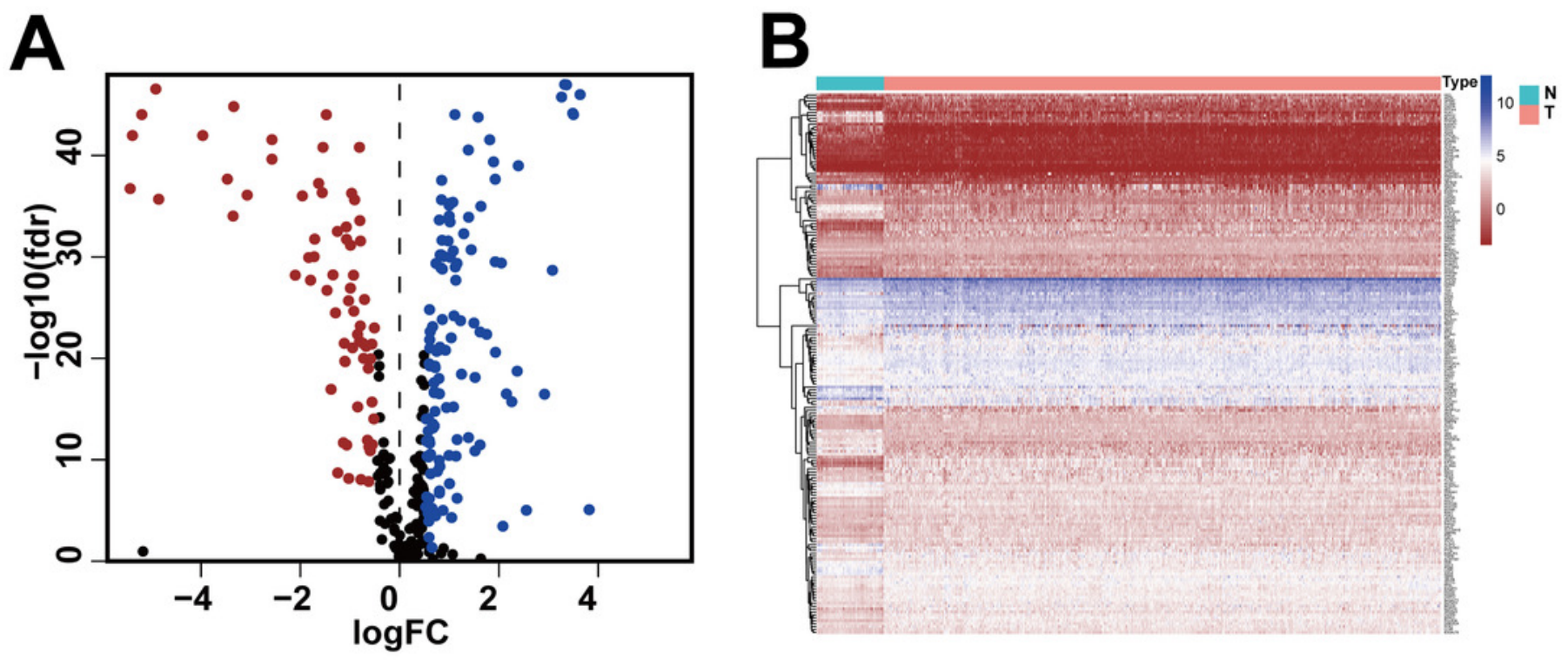
Figure 2

Screening DGRG with prognostic value.

(A) Based on univariate Cox regression, the forest map showed that there were 14 DGRGs with prognostic significance $(P<0.05)$. $(B, C)$ LASSO regression further screened out 13 DGRGs that were closely related to the prognosis.
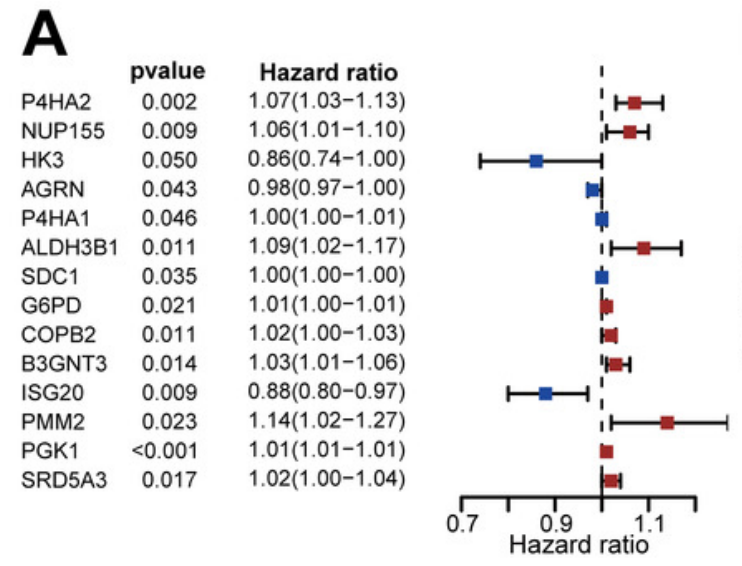

B

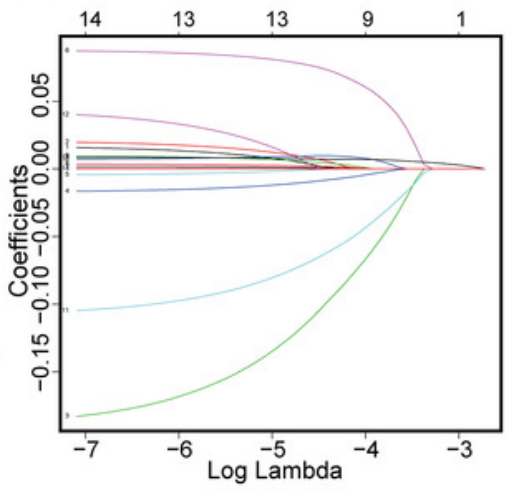

C

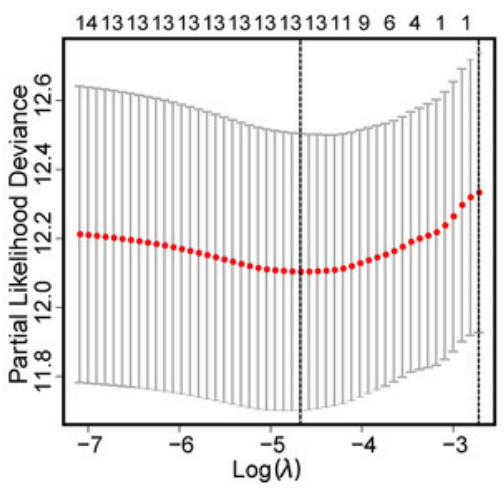




\section{Figure 3}

Construction of the risk-scoring model for patients with breast invasive ductal carcinoma based on DGRG.

(A) Kaplan-Meier survival analysis showed that patients in the high-risk group had a shorter OS than that of the low-risk group $\left(P=P=9.795 \times 10^{-8}\right)$. (B) The ROC curve showed that the AUC of the 3-year OS and 5-year OS were 0.755 and 0.726 , respectively. (C-E) The overview of survival time for each patient, the distributions of risk scores for each patient and heatmaps of expression profiles for 13-DGRG between the low-risk group and the high-risk group.
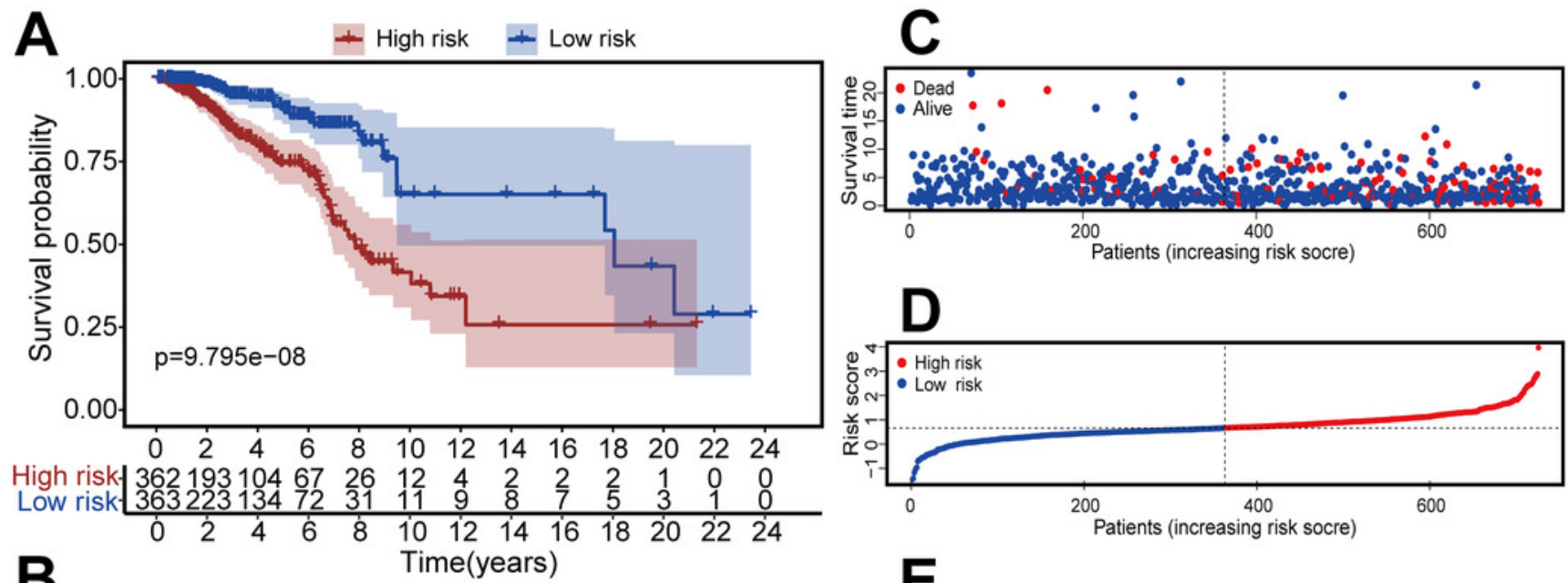

B
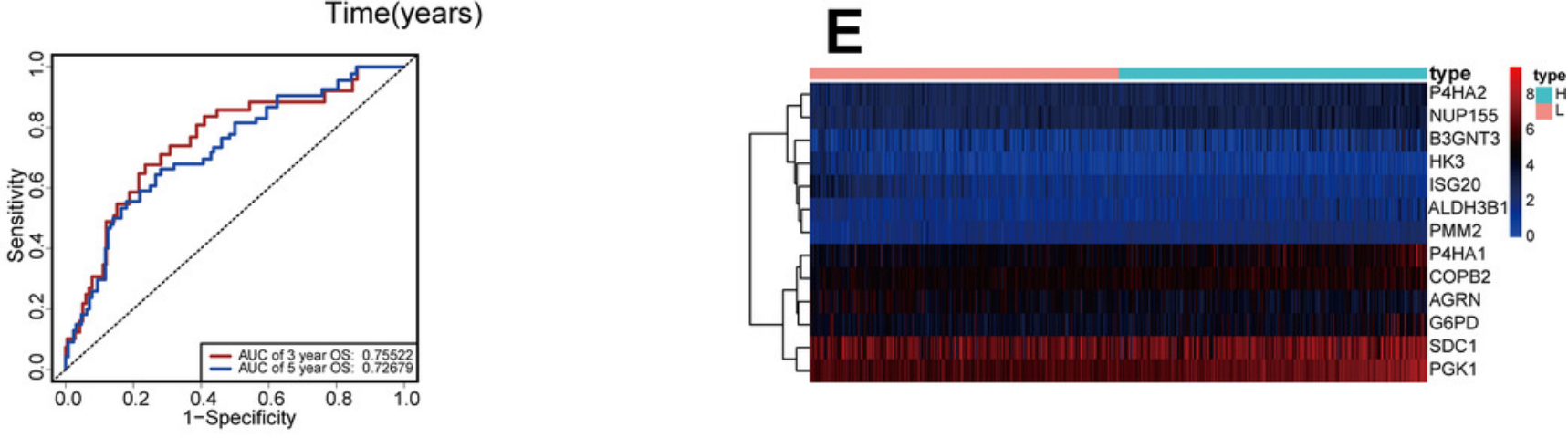


\section{Figure 4}

Assessment of risk scores and prognostic value of clinical data.

(A) Univariate Cox analysis showed that risk scores and clinical variables including age, TMN stage, and whether it was TNBC or not were significantly related to OS. (B) Multivariate Cox analysis manifested that the 13-DGRG signature was an independent prognostic indicator for patients with breast invasive ductal carcinoma.

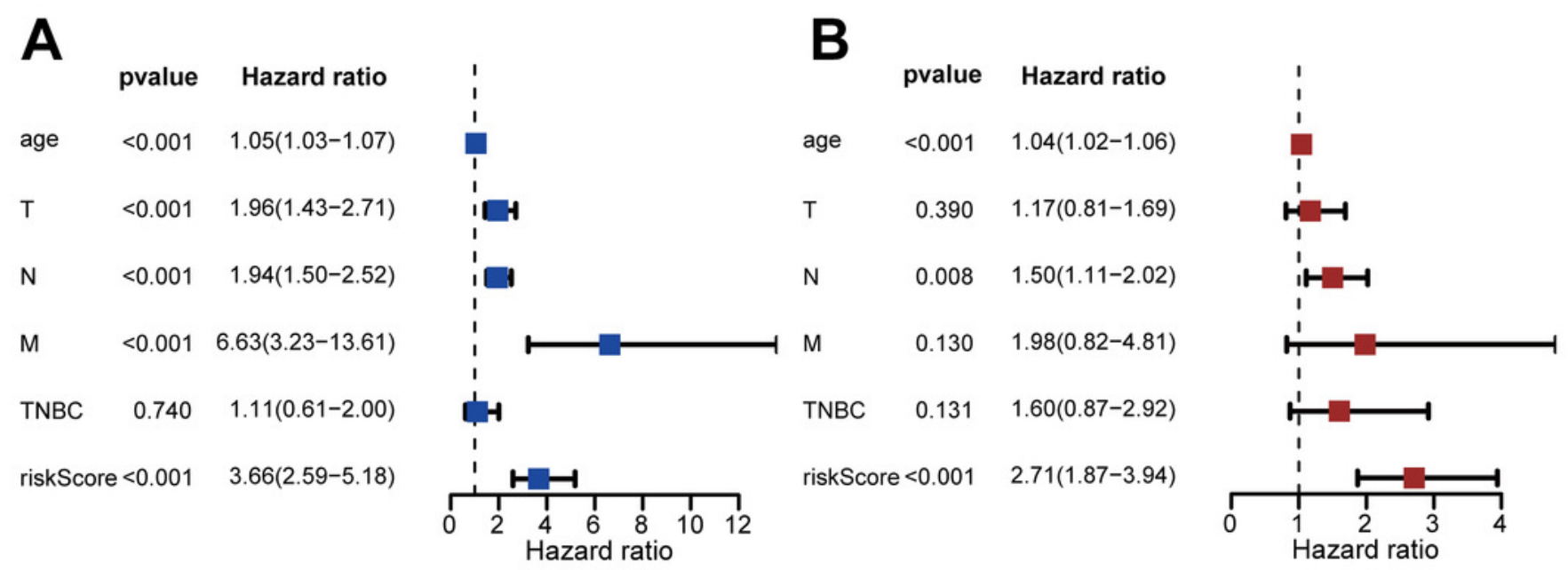




\section{Figure 5}

Clinical survival analysis of subgroups' DGRG risk scores for breast invasive ductal carcinoma.

(A) In the T1-T2 subgroup, the OS of the high-risk group was lower than that of the low-risk group ( $\mathrm{P}<0.001)$. (B) In the T3-T4 subgroup, the OS of the high-risk group was lower than that of the low-risk group $(P=0.006)$. (C) In the NO subgroup, the OS of the high-risk group was lower than that of the low-risk group $(P<0.001)$. (D) In the N0-N3 subgroup, the OS of the high-risk group was lower than that of the low-risk group $(P=0.006)$. (E) In the M0 subgroup, the OS of the high-risk group was lower than that of the low-risk group $(P<0.001)$. (F) In the M1 subgroup, there was no significant difference in OS between patients in the high-risk group and the low-risk group, because the number of cases in the M1 subgroup is relatively small $(P=0.857)$. $(G)$ In the TNBC subgroup, the OS of the high-risk group was lower than that of the low-risk group $(\mathrm{P}<0.001)$. $(\mathrm{H})$ In the NTNBC subgroup, the OS of the high-risk group was lower than that of the low-risk group $(P=0.007)$. The above results suggested that the DGRG risk-scoring model had a good predictive ability. 


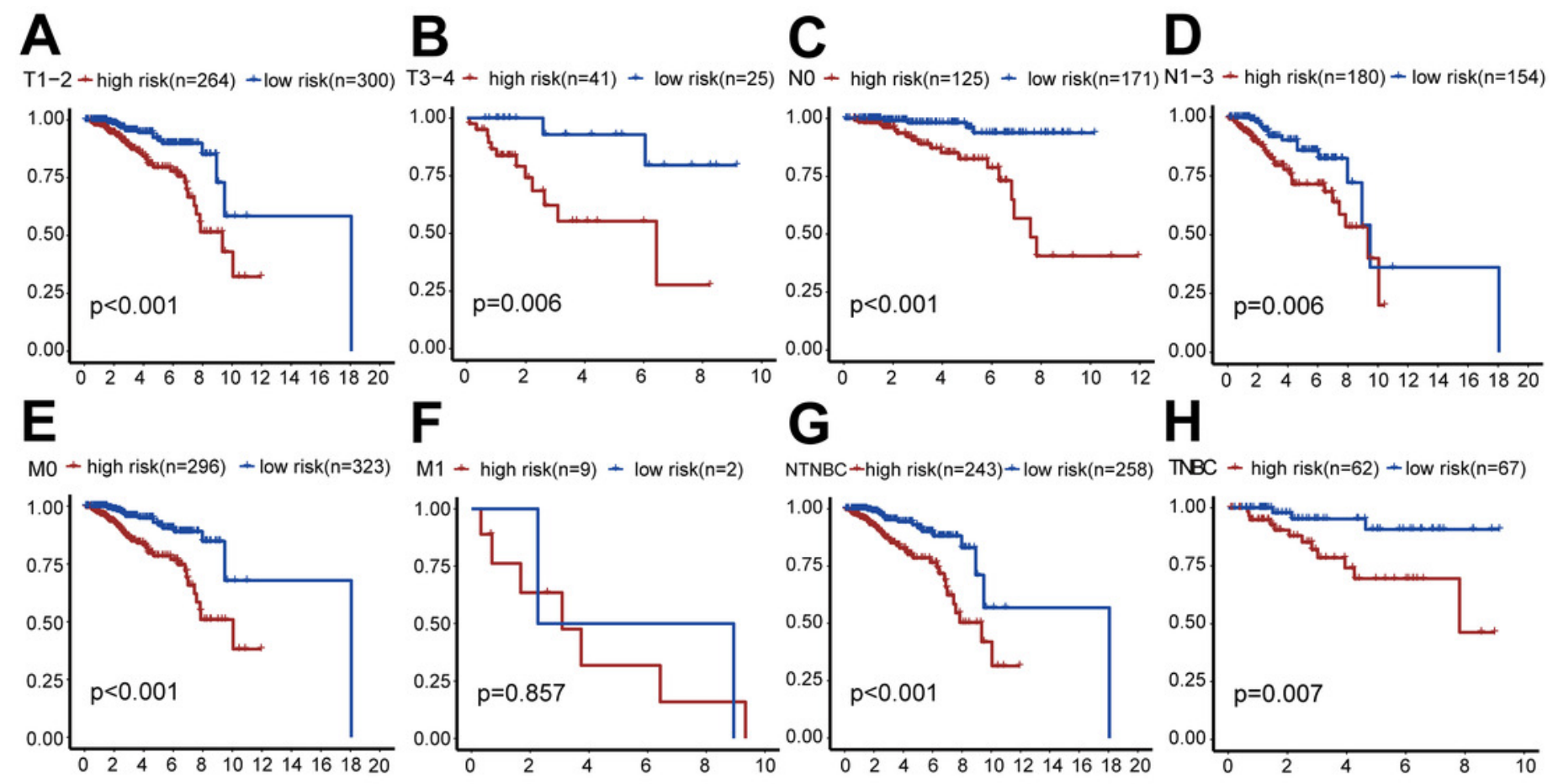


Figure 6

External validation of the risk scoring model The model was verified in GEO datasets (GSE131769).

Kaplan-Meier survival curves showed that the OS of patients with high-risk was significantly shorter than in the low-risk group $\left(P=P=3.245 \times 10^{-3}\right.$, Figure $\left.6 \mathrm{~A}\right)$. The ROC curve was drawn to calculate the AUC of the 3-year OS and 5-year OS as 0.731 and 0.728 , respectively (Figure 6B).

$\mathbf{A}$

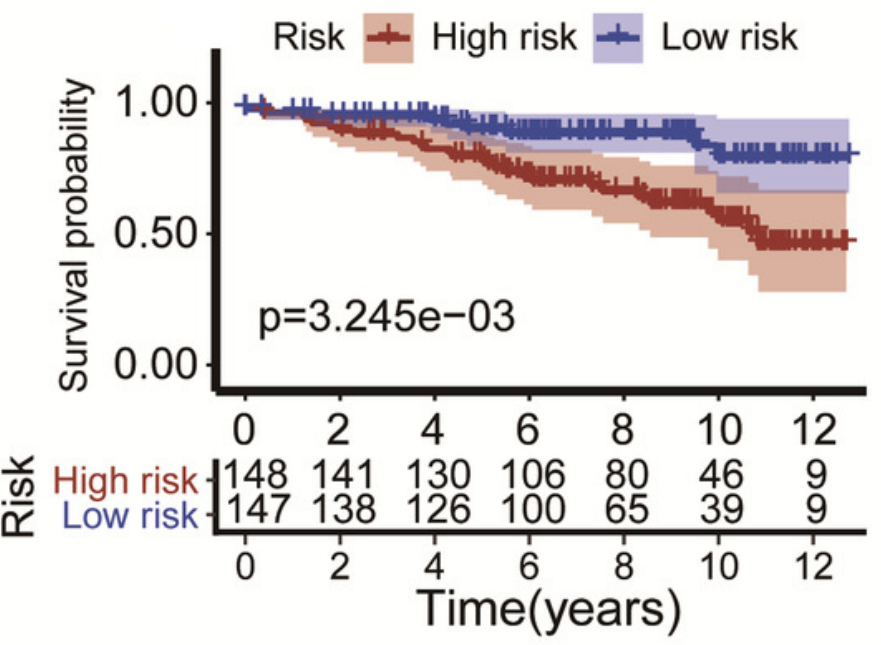

B

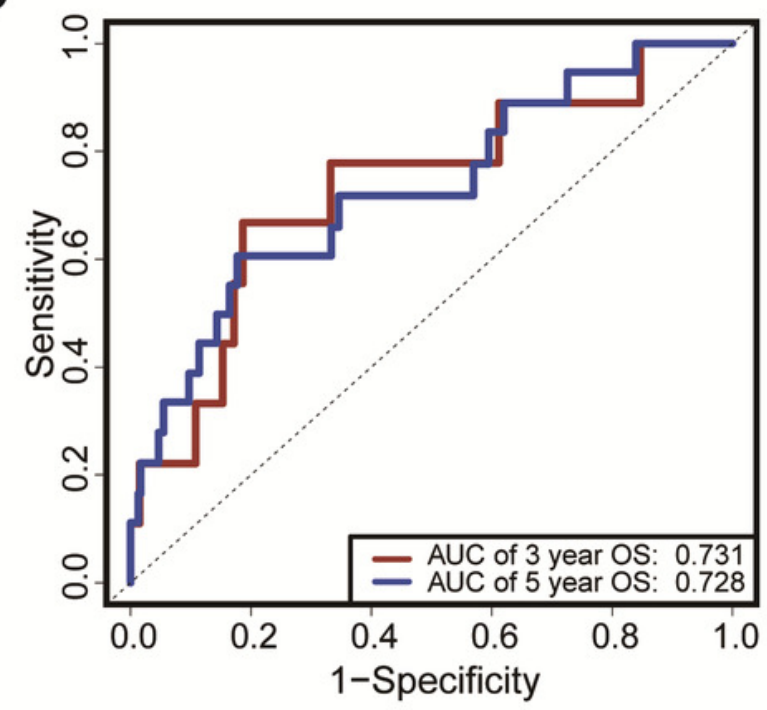


Figure 7

Establishment and evaluation of the nomogram.

(A) The nomograms for predicting the patients' OS. (B) ROC curve analysis showed that AUC of 3-year and 5-year OS were 0.842 and 0.808 , respectively. (C) The calibration curve for the 3-year OS of the nomogram. (D) The calibration curve for the 5-year OS of the nomogram. (E) The clinical decision curve for the 3-year OS of the nomogram. (F) The clinical decision curve for the 5-year OS of the nomogram.

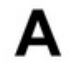

Points

age

$\mathrm{T}$

N

M

TNBC

riskScore

Total Points

3-year survival

5-year survival

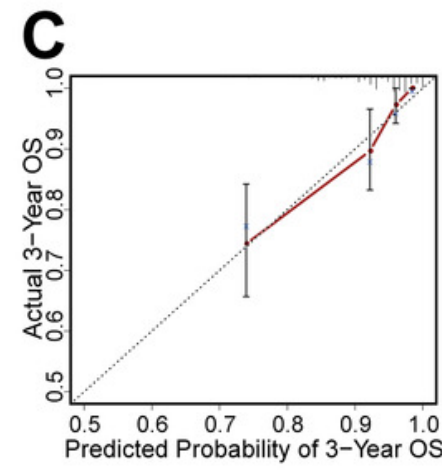

$\overbrace{\mathrm{T} 1-2}^{\mathrm{T} 1-3}$

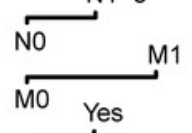

No Yes $\begin{array}{lllllllllll}0 & 10 & 20 & 30 & 40 & 50 & 60 & 70 & 80 & 90 & 100\end{array}$

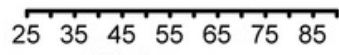

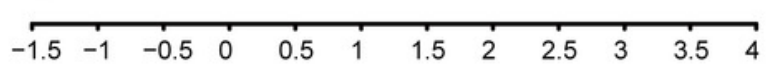

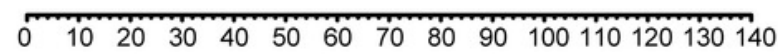
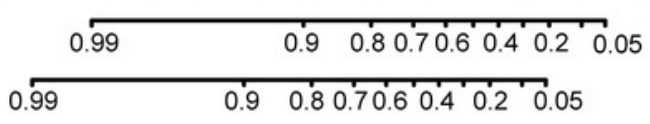

\section{D}

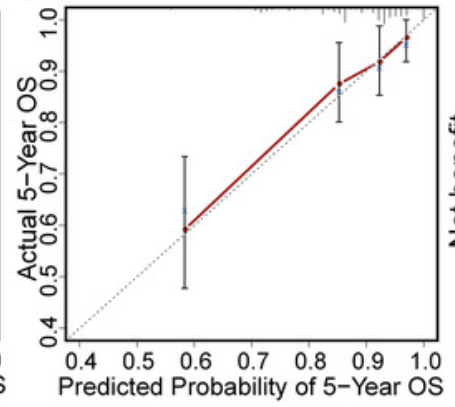

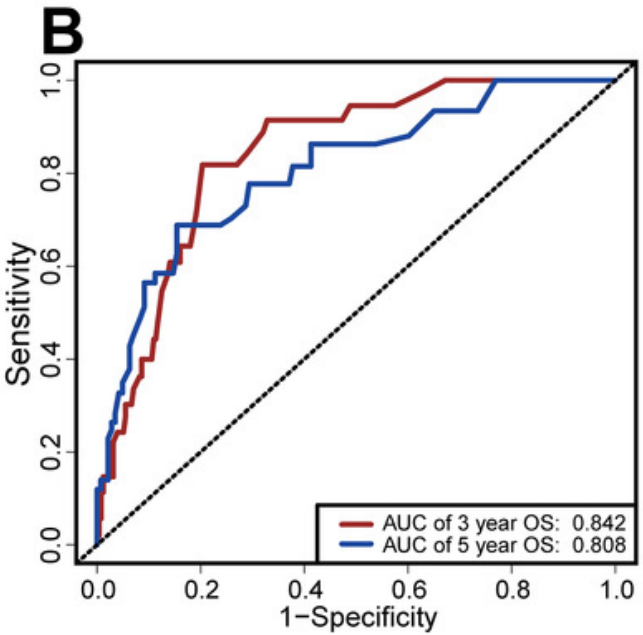
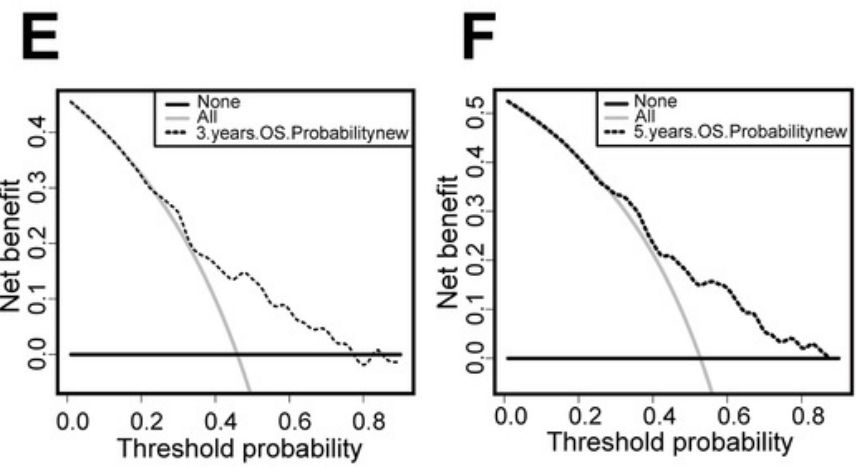


\section{Figure 8}

Enrichment analysis.

(A-H) KEGG pathway analysis showed that these genes were involved in cell cycle, DNA replication, glycolysis gluconeogenesis, RNA degradation, arachidonic acid metabolism, cytokine cytokine receptor interaction, cytosolic DNA sensing pathway and ribosome. (I-P) GO enrichment analysis showed that the genes were enriched in the cell cycle G1-S phase transition, DNA geometric change, meiotic cell cycle process, regulation of cellular response to heat, cytokine mediated signaling pathway, regulation of homotypic cell cell adhesion, regulation of production of molecular mediator of immune response, $T$ cell differentiation. 

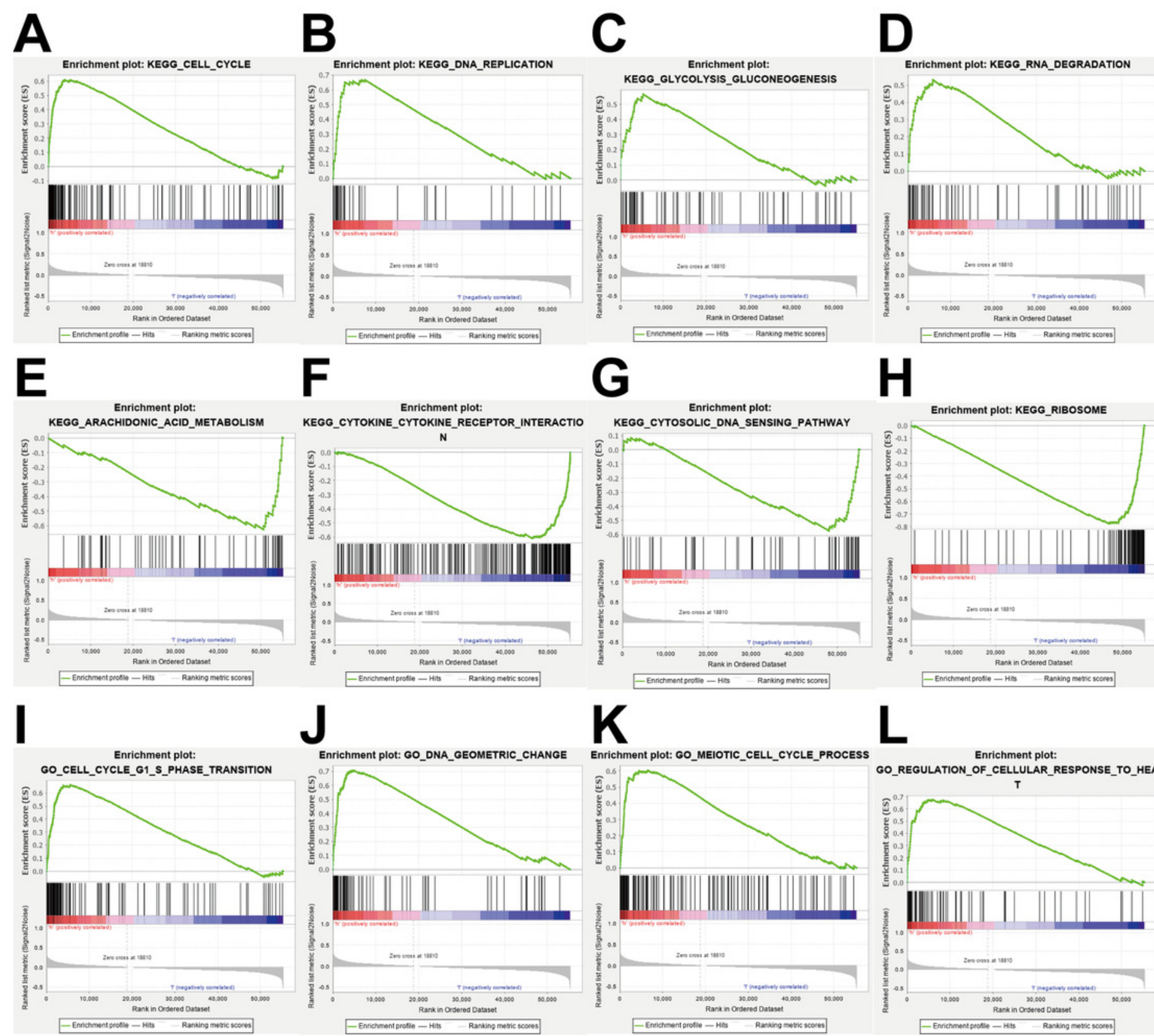

H

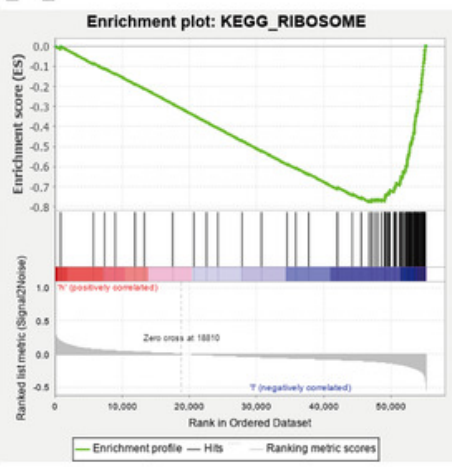

M

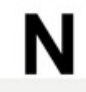

Enrichment plot:

0

Enrichment plot:
Enrichment plot:
GO_REGULATION_OF_HOMOTYPIC_CELL_CELL_ADHES GO_REGULATION_OF_PRODUCTION_OF_MOLECULAR_

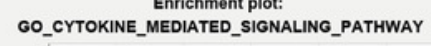

\section{年}
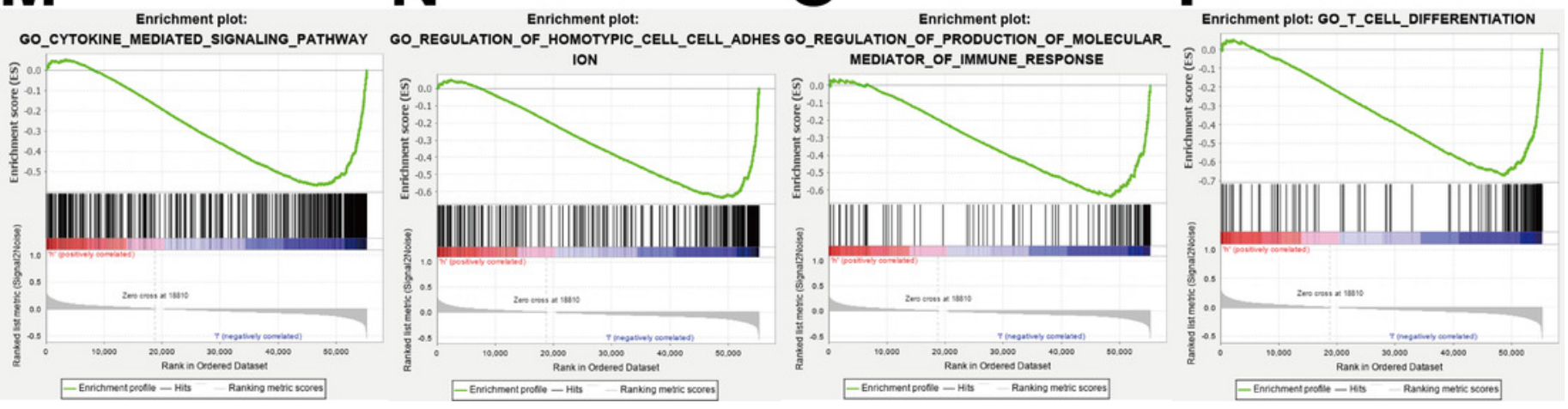
Figure 9

Construction of a network diagram of the interaction between DGRG

(A) The analysis of STRING showed that there were interactions among 6 genes (P4HA2, P4HA1, PGK1, G6PD, HK3, PMM2) in DGRG.

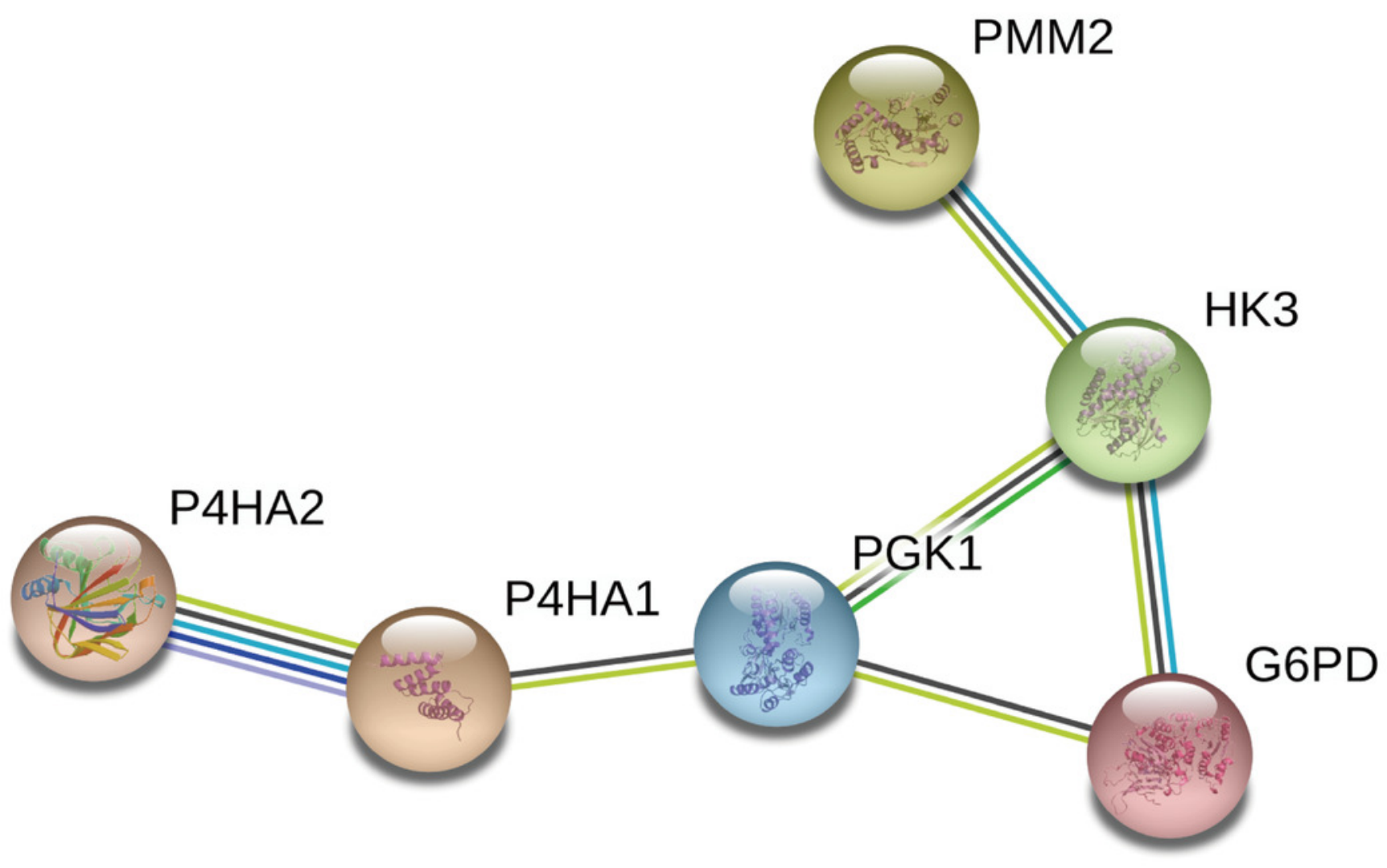

